\title{
Microstructures to Control Elasticity in 3D Printing
}

\author{
Christian Schumacher ${ }^{1,2} \quad$ Bernd Bickel $^{1,3} \quad$ Jan Rys $^{2} \quad$ Steve Marschner $^{4} \quad$ Chiara Daraio $^{2} \quad$ Markus Gross $^{1,2}$ \\ ${ }^{1}$ Disney Research Zurich $\quad{ }^{2}$ ETH Zurich $\quad{ }^{3}$ IST Austria $\quad{ }^{4}$ Cornell University
}

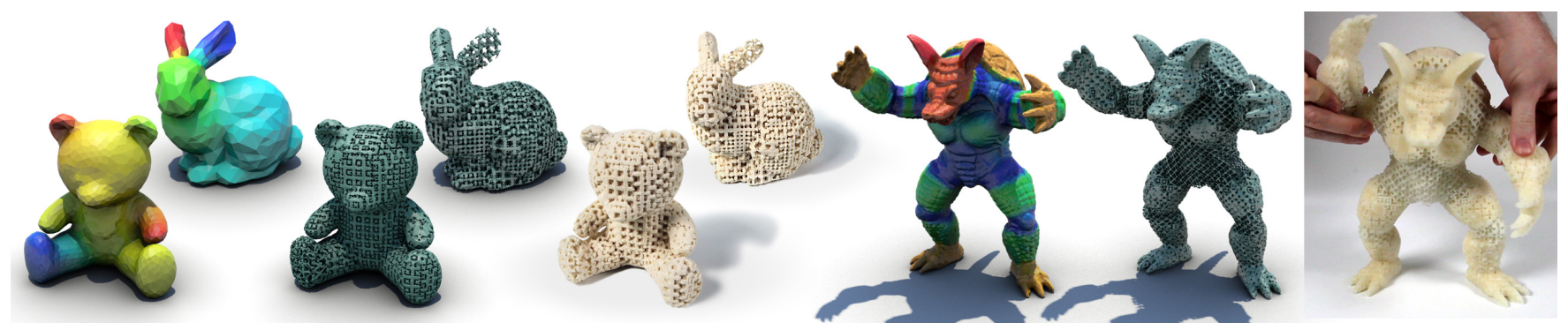

Figure 1: Given a virtual object with specified elasticity material parameters (blue=soft, red=stiff), our method computes an assemblage of small-scale structures that approximates the desired elastic behavior and requires only a single material for fabrication.

\begin{abstract}
We propose a method for fabricating deformable objects with spatially varying elasticity using $3 \mathrm{D}$ printing. Using a single, relatively stiff printer material, our method designs an assembly of smallscale microstructures that have the effect of a softer material at the object scale, with properties depending on the microstructure used in each part of the object. We build on work in the area of metamaterials, using numerical optimization to design tiled microstructures with desired properties, but with the key difference that our method designs families of related structures that can be interpolated to smoothly vary the material properties over a wide range. To create an object with spatially varying elastic properties, we tile the object's interior with microstructures drawn from these families, generating a different microstructure for each cell using an efficient algorithm to select compatible structures for neighboring cells. We show results computed for both 2D and 3D objects, validating several $2 \mathrm{D}$ and $3 \mathrm{D}$ printed structures using standard material tests as well as demonstrating various example applications.
\end{abstract}

CR Categories: I.3.5 [Computer Graphics]: Computational Geometry and Object Modeling-Physically based modeling

Keywords: fabrication, topology optimization, 3D printing

\section{Introduction}

With the emergence of affordable 3D printing hardware and online 3D printing services, additive manufacturing technology comes with the promise to make the creation of complex functional physical artifacts as easy as providing a virtual description. Many functional objects in our everyday life consist of elastic, deformable material, and the material properties are often inextricably linked to function. Unfortunately, elastic properties are not as easy to control as geometry, since additive manufacturing technologies can usually use only a single material, or a very small set of materials, which often do not match the desired elastic deformation behavior. However, 3D printing easily creates complex, high-resolution 3D structures, enabling the creation of metamaterials with properties that are otherwise unachievable with available printer materials.

Metamaterials are assemblies of small-scale structures that obtain their bulk properties from the shape and arrangement of the structures rather than from the composition of the material itself. For example, based on this principle, Lakes [1987] presented the first engineered materials that exhibit a negative Poisson's ratio. Since then, numerous designs have been proposed, usually consisting of a periodic tiling of a basic pattern, and engineering their structures is an active area of research [Lee et al. 2012].

While designing a tiled microstructure to match given homogeneous material properties can be achieved with modest extensions to the state of the art, designing a complex microstructural assembly to achieve heterogeneous, spatially varying properties is much more challenging. We face a complex inverse problem: to determine a discrete small-scale material distribution at the resolution of the 3D printer that yields the desired macroscopic elastic behavior. Inverse problems of this type have been explored for designing periodic structures that can be tiled to synthesize homogeneous volumes, but the methods are computation-intensive and do not scale to designing non-periodic structures for objects with spatially varying material properties.

Our goal is to enable users to employ metamaterials in their 3D printing workflow, generating appropriate structures specifically for their available 3D printer model and base material within seconds. Clearly, designing entire models on the fly by directly optimizing over the whole structure is impractical. Instead, we propose a data-driven approach that efficiently assembles models out of precomputed small-scale structures so that the result both resembles the desired local elasticity and also is within the capabilities of the available output device.

First, we precompute a database of tiled structures indexed by their elastic properties. We want these structures to cover a large and ideally continuous region in the space of possible elastic behaviors. To achieve this goal, we introduce an optimization method for sampling structures that exhibit a range of desired behaviors, but are 
also sufficiently similar to allow interpolation. We initialize this optimization method either with a known structure or desired elastic property values, and then compute a family of similar structures that covers a subspace of possible elastic behaviors. We repeat this process, each time adding a new family of structures, to increase the coverage of the material space.

Second, to synthesize the metamaterials for a specific object, we tile the object's interior with microstructures drawn from these families. Note that the same elastic behavior can be reproduced by structures that differ significantly in shape, and therefore the families might overlap in material space. This is an important feature of our system for reproducing spatially varying material behavior. Spatially neighboring structures must connect properly at their interface, and overlapping families provide us with a set of candidates for each tile, significantly increasing the chance of finding structures compatible with neighboring tiles. We propose an efficient global optimization algorithm that selects an optimized tiling, taking into account the need for neighboring structures to connect properly. Our final result is functional and $3 \mathrm{D}$ printable.

We evaluate our algorithm by fabricating several examples of both flat sheets and 3D objects with heterogeneous material behavior. For several isotropic and anisotropic 2D examples and isotropic 3D examples, we measure the resulting elastic properties, comparing the actual material parameters to the values predicted by our simulation.

\section{Related Work}

Simulation and Homogenization Simulation of deformable objects has a long history in computer graphics [Nealen et al. 2006]. For accurate simulation of material behavior, the finite element method is a popular choice, with a wide range of available constitutive models of materials. An excellent introduction can be found in Sifakis and Barbič [2012]. Inspired by the seminal work of Hashin and Shtrikman [1963], homogenization theory was developed to efficiently simulate inhomogeneous materials with fine structures, allowing microscopic behavior to be averaged into a coarser macroscopic representation with equivalent behavior at the macroscopic scale [Michel et al. 1999; Cioranescu and Donato 2000]. Nesme et al. [2009] encode the material stiffness within coarse elements using shape functions after a fine-level static analysis. We build on the numerical coarsening approach by Kharevych et al. [2009] which turns the heterogeneous elastic properties represented by a fine mesh into possibly anisotropic elastic properties of a coarse mesh that effectively captures the same physical behavior. After computing harmonic displacements to capture how the fine mesh behaves, their approach presents an analytic relationship between the elasticity tensors of a coarse element and the elasticity tensors of the fine elements contained within. We extend this formulation for inverse homogenization.

Mechanical Metamaterials and Inverse Homogenization Metamaterials are usually defined as macroscopic composites having a manmade, periodic cellular architecture designed to produce a behavior not available in nature. In this paper, we draw inspiration from mechanical metamaterials, and relax the term in the context of 3D printing to material properties not available on 3D printers. Lakes [1987] presented the first engineered materials that exhibit a negative Poisson's ratio. Due to their structure, these materials expand laterally when stretched, therefore increasing their volume. Since then, numerous designs for soft metamaterials have been proposed, either found by intuition, or numerical optimization processes [Lee et al. 2012].

In classical inverse homogenization approaches the goal is to find a repetitive small-scale structure with desired macroscopic properties. This is obtained by optimizing the material distribution in the base cell. Researchers have proposed various parametrizations of the material distribution, such as networks of bending beams [Hughes et al. 2010], spherical shells patterned with an array of circular voids [Babaee et al. 2013], or rigid units [Attard and Grima 2012]. Alternatively, the domain of a base cell can be discretized into small material voxels, and a discrete value problem has to be solved. Due to the combinatorial complexity, direct search methods are prohibitively expensive, and the problem is usually solved using a relaxed formulation with continuous material density variables [Sigmund 2009] or advanced search heuristics [Huang et al. 2011]. These approaches generally search for structures with extreme properties, often maximum stiffness, for a given amount of material, and only consider a single structure. In contrast, we present an optimization method that computes a structure to achieve a specific material behavior. Based on this method, we span an entire space of elastic material structures, and construct a mapping from elasticity parameters to microstructures that can be efficiently evaluated during runtime.

Rodrigues et al. [2002] and Coelho et al. [2008] suggest methods for hierarchical topology optimization, computing a continuous material distribution on a coarse level and matching microstructures for each coarse cell. While in their approach each microstructure cell can be optimized independently, each of them still needs to be computed based on a costly optimization scheme, and there is no guarantee on the connectivity of neighboring structures. In contrast, we use a data-driven approach which allows us to synthesize structures extremely efficiently, and also take the quality of the connectivity into account. For functionally graded materials with microstructures, Zhou et al. [2008] guarantee the matching of boundaries either by prescribing connectors or by incorporating a complete row of cells that form a gradient during a single optimization. We follow a different strategy. Instead of restricting types of connections or increasing the size of structures, we efficiently compute multiple candidates from families of microstructures and then select structures with interfaces that match best.

Fabrication-Oriented Material Design In computer graphics, we are currently witnessing an increasing interest in fabricationoriented material design for reproducing 3D physical artifacts from virtual representations. Recently, Chen et al. [2013] presented an abstraction mechanism for translating functional specifications to fabricable 3D prints, and Vidimče et al. [2013] introduced a programmable pipeline for procedural evaluation of geometric detail and material composition, allowing models to be specified easily and efficiently. For static objects, Zhou et al. [2013] present an algorithm for efficiently analyzing the structural strength, and Stava et al. [Stava et al. 2012] improve the structural strength by automatic hollowing, thickening, and strut insertion. Wang et al. [Wang et al. 2013] propose a method for computing skin-frame structures for the purpose of reducing the material cost of the printed object.

Recent work also investigated the reproduction of appearance, for example by modulating the surface structure to achieve desired reflection properties [Weyrich et al. 2009; Lan et al. 2013; Rouiller et al. 2013], by interleaving different colored materials on the surface [Reiner et al. 2014], or by volumetric combination of multiple materials [Hašan et al. 2010; Dong et al. 2010] to control subsurface scattering behavior. Conceptually similar to our approach, these methods are based on the principle that the large-scale appearance is governed by small-scale details, and can reproduce appearance properties which are significantly different from the 3D printer's base material.

Bickel et al. [2010] presented a data-driven process for designing 

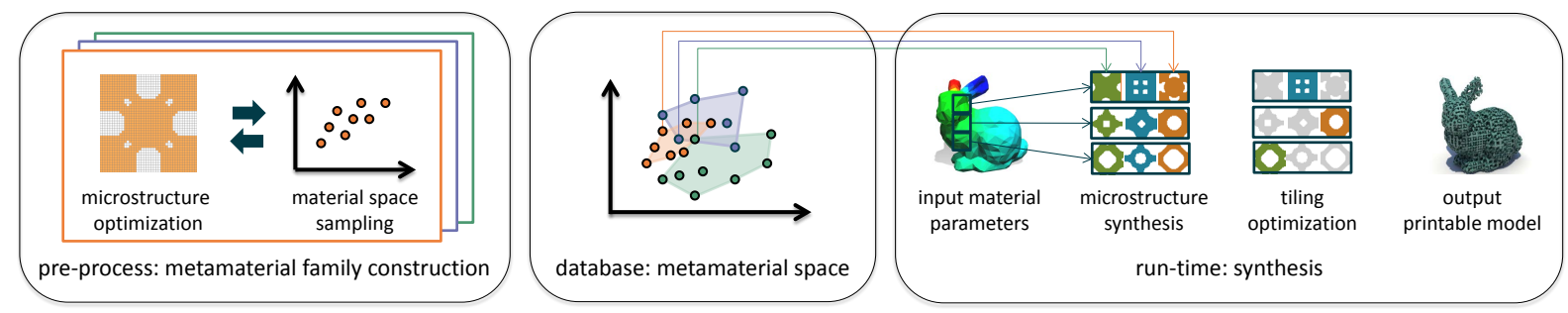

Figure 2: An overview of our system. In a pre-processing step, we compute metamaterial families. Each family consists of multiple related microstructures that can be interpolated to smoothly vary the material properties. We store all families in a database, representing our metamaterial space. Given as input an object with specified material parameters, we synthesize locally microstructures that resemble the desired deformation behavior. As multiple, topologically different structures can have the same bulk behavior, we potentially have multiple candidates of microstructures for a single location. Using these candidates, we compute an optimized tiling, ensuring that neighboring structures are properly connected. Finally, the physical prototype is $3 D$ printed.

and fabricating objects with desired deformation behavior. Whereas Bickel et al. start from specified example deformations, the input to our system is a model with specified elastic parameters. Their approach assumes that a small set of microstructures is already given, and finds a discrete combination of stacked layers from these structures that meets the criteria specified by the example deformations. In contrast, our work computes the small-scale structures themselves. This is essential for more precise local control of the deformation. Furthermore, Bickel et al. select a material for each layer using a branch-and-bound discrete optimization operating on the exponential space of designs, requiring running times on the order of an hour for examples with five layers and nine base materials. By contrast, our method synthesizes the desired structures from pre-computed continuous material subspaces, and is able to handle objects with thousands of layers or cells within seconds.

Several previous methods investigate fitting spatially varying material parameters either from measurements of real-world objects [Becker and Teschner 2007], infer them from user-specified input such as example deformation [Skouras et al. 2013], or optimize material distributions to achieve higher-level functionality such as locomotion of soft robots [Hiller and Lipson 2012]. Recently, Xu et al. [2015] presented an interactive material design tool, which computes a spatial distribution of material properties given user-provided displacements and forces at a set of mesh vertices. Our method complements these approaches, working towards the goal of automatically converting the virtual representation obtained by those methods into 3D printable objects.

\section{Overview}

The goal of our system is to automatically convert an object with given spatially varying elastic properties into a $3 \mathrm{D}$ printable representation that requires only a single base material for fabrication, and mimics the desired elastic behavior. In this work, we limit ourselves to small strains and demonstrate our approach for both isotropic and anisotropic elastic materials. As outlined in Figure 2, our system consists of two main stages: a preprocessing step that constructs metamaterial structures covering the space of reproducible material properties, and a synthesis stage that uses those structures to generate microstructures for a given object. We start the description by defining the most important terms we use throughout the paper.

Metamaterial Space A metamaterial space is a specific organization of metamaterials. We target reproducing elastic behavior using $3 \mathrm{D}$ printers and represent their behavior using the $n$ parameters of the underlying constitutive model. For example, linear isotropic materials are represented by $n=2$ parameters, the Young's mod- ulus and Poisson's ratio. In addition, every metamaterial space has a mapping function, mapping the $n$ parameters to one or several microstructures. This mapping is one-to-many because different microstructures might reproduce the same elastic behavior.

Metamaterial Space Construction Our first stage aims to define a function that efficiently maps a given elastic material to a small, tileable structure with the same bulk behavior. Our process starts by systematically sampling the material parameter space. Our strategy is to compute a sparse set of representative structures that (i) cover a wide range in the space of elastic material parameters and that (ii) allow the interpolation of neighboring structures in parameter space. Via a weighted combination of these samples, we then reconstruct a continuous mapping from elasticity parameters to microstructures. We call such a set of interpolatable structures a metamaterial family. A metamaterial family defines a one-to-one mapping from material properties to structure. In practice, a family usually only covers a partial gamut of the material space. Therefore, we pre-compute several families until we sufficiently cover a desired range of elastic behavior. All metamaterial families together constitute our metamaterial space. Note that, as shown in Figure 10, the gamut of these metamaterial families might be partially overlapping, yielding a one-to-many mapping from parameters to microstructures.

Synthesis To synthesize the microstructure for a given object, we tile its interior. For each tile, we interpolate a microstructure from each pre-computed family of structures. This provides us with a small set of candidate structures for each cell, out of which we have to select exactly one. These choices are not independent; they must be made consistently so that the structures connect well with their neighboring tiles. We suggest a carefully designed metric that quantifies the compatibility of structures and phrase this selection as a combinatorial problem, which we solve efficiently using an optimization method based on message passing. Finally, we use the selected structures to fabricate the object using rapid prototyping.

\section{Background}

In order to determine and optimize for the behavior of a microstructure, a physical model is introduced. This model combines standard linear elasticity with extensions specific to microstructure simulation and topology optimization.

\subsection{Linear Elasticity}

Continuum mechanics in combination with a finite elements discretization is a popular way to model elastic structures. For stan- 
dard linear elasticity, the energy density can be defined as

$$
W=\varepsilon: \mathbf{C}: \varepsilon=\varepsilon: \boldsymbol{\sigma} .
$$

Here, $\varepsilon$ is the linear Cauchy strain, $\mathbf{C}$ is the material stiffness tensor, and $\boldsymbol{\sigma}=\mathbf{C}: \varepsilon$ is the Cauchy stress tensor. The material stiffness tensor is defined by a small set of parameters that depend on the material model. For example, for an isotropic material, it is a function of 2 parameters, the Young's modulus and the Poisson's ratio. For more complex materials, additional parameters such as the shear modulus and direction-dependent Young's moduli and Poisson's ratios are used. Given parameters $\mathbf{p}$, we denote the corresponding material stiffness tensor as $\mathbf{C}(\mathbf{p})$.

A linear finite element method is used to discretize (1) with basis functions defined on triangular and tetrahedral elements in 2D and $3 \mathrm{D}$, respectively. In this case, the strain $\varepsilon_{i}$ and material stiffness tensor $\mathbf{C}_{i}$ are constant within an element $i$. The elastic energy, given as the volume integral of the energy density, is then

$$
U_{e l}=\sum_{i=1}^{k}\left(\boldsymbol{\varepsilon}_{i}: \mathbf{C}_{i}: \boldsymbol{\varepsilon}_{i}\right) V_{i}
$$

for a model with $k$ elements and element areas/volumes $V_{i}$. The total energy of the system also considers external forces and tractions. Summarizing surface traction and forces using a general force field f acting on vertices, this energy can be expressed as

$$
U_{\text {tot }}(\mathbf{x})=U_{e l}(\mathbf{x})-\sum_{i=1}^{n} \mathbf{x}_{i}^{T} \mathbf{f}_{i}
$$

where $n$ is the number of vertices, and vector $\mathbf{x}=\left[\mathbf{x}_{1}^{T} \cdots \mathbf{x}_{n}^{T}\right]^{T}$ is the concatenation of all vertex position vectors. The deformed configuration $\mathbf{x}$ corresponding to the static equilibrium can be computed by minimizing this energy, or equivalently, solving

$$
\nabla_{\mathbf{x}} U_{e l}(\mathbf{x})=\mathbf{f}
$$

Since the elastic energy $U_{e l}(\mathbf{x})$ is invariant to translation and rotation, the solution to this problem is not unique. A common workaround to this is to constrain enough degrees of freedom to get rid of this nullspace. However, the choice of degrees of freedom might influence the solution in the presence of forces. Instead, we opt to resolve the ambiguities by introducing constraints on the moments of the object, similar to Zhou et al. [2013]. These constraints take the form

$$
\begin{aligned}
& \mathbf{c}_{1}(\mathbf{x})=\sum_{i=1}^{n}\left(\mathbf{x}_{i}-\mathbf{X}_{i}\right)=0 \\
& \mathbf{c}_{2}(\mathbf{x})=\sum_{i=1}^{n}\left(\left(\mathbf{x}_{i}-\mathbf{X}_{i}\right) \times\left(\mathbf{x}_{i}-\overline{\mathbf{X}}\right)\right)=0
\end{aligned}
$$

where $\mathbf{X}_{i}$ is the rest state position of vertex $i$, and $\overline{\mathbf{X}}$ is the mean rest state position. For simplicity, we combine these constraints into a single vector $\mathbf{c}(\mathbf{x})=\left[\mathbf{c}_{1}(\mathbf{x})^{T} \mathbf{c}_{2}(\mathbf{x})^{T}\right]^{T}$. Intuitively, these constraints fix the mean translation and rotation. To compute $\mathbf{c}_{2}$ in the $2 \mathrm{D}$ case, we treat positions as points on the $z=0$ plane and use the $z$-component of the cross product.
Combining Equation (4) and Equation (5), the problem of computing the equilibrium configuration then becomes

$$
\nabla_{\mathbf{x}} U_{e l}(\mathbf{x})=\mathbf{f} \quad \text { s.t. } \quad \mathbf{c}(\mathbf{x})=\mathbf{0} .
$$

This problem can be expressed as a system of linear equations and solved efficiently.

\subsection{Microstructure Simulation}

The simulation of a microstructure can be simplified by considering a tiling of a representative base cell. Instead of simulating the complete tiling to determine the behavior of the structure, periodic boundary conditions can be added to a simulation of a single cell. These boundary conditions simulate the tiling and essentially require that opposite boundaries of the cell have the same shape.

Specifically, we assume that any vertex on a boundary has a matching vertex on the opposite boundary such that its relative position on the boundary is identical. Choosing an arbitrary pair of boundary vertices $\mathbf{x}_{0}^{\text {base }}$ and $\mathbf{x}_{1}^{\text {base }}$ as base vertices then defines the distance between two opposite boundaries, and any other vertex $\mathbf{x}_{j}$ on one boundary can be expressed as a combination of the base vertices and the corresponding vertex $\mathbf{x}_{i}$ on the opposite

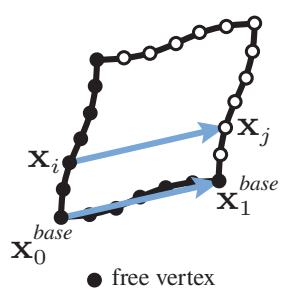

- free vertex o constrained vertex boundary [Smit et al. 1998]

$$
\mathbf{x}_{j}=\mathbf{x}_{1}^{\text {base }}+\mathbf{x}_{i}-\mathbf{x}_{0}^{\text {base }} .
$$

These boundary conditions can be efficiently integrated into a simulation by removing the corresponding vertices from the degrees of freedom.

\subsection{Numerical Coarsening}

Optimizing a microstructure is an inverse problem, corresponding to the forward problem of determining the coarse-scale behavior from the microstructure. This forward problem can be defined using the idea of homogenization: compute a material stiffness tensor for a homogeneous material whose elastic behavior matches that of the tiled microstructure. We use the Numerical Coarsening approach [Kharevych et al. 2009], which uses a set of load cases to approximate the coarse elastic behavior of a given structure. Essentially, given the deformations $\mathbf{h}$ that these load cases induce, which are called harmonic displacements, the method computes a single material stiffness tensor $\mathbb{C}(\mathbf{h})$ that describes the homogenized material behavior of a microstructure, which we will use to solve the inverse problem. We refer to the supplemental material for a detailed introduction to the Numerical Coarsening approach.

\section{Microstructure Optimization}

Our microstructure optimization method solves the inverse problem to the Numerical Coarsening method mentioned in the previous section, solving for a microstructure that coarsens to a given stiffness tensor.

Optimizing a microstructure requires a way to define and alter the material distribution within a cell. A common approach in topology optimization is to discretize the material distribution by subdividing the cell into a grid of material voxels [Sigmund 2009], where each voxel is associated with a binary activation that describes 
whether the voxel is full (1) or void (0). However, optimizing the microstructure using these binary variables directly would be infeasible for moderately large grids. Instead, the problem is usually relaxed by allowing the activations to vary smoothly between 0 and 1 during the optimization, and only requiring them to converge to a binary solution at the end of the optimization. For the continuous activations, a meaningful interpolation between void and full voxels has to be defined such that the activation corresponds to a physical quantity in the simulation. A simple way to define this is by interpolating between stiffness tensors. For any voxel $i(1 \leq i \leq m$, with $m$ being the number of voxels), an individual material stiffness tensor $\mathbf{C}_{i}$ is defined as an interpolation between the base material stiffness tensor $\mathbf{C}_{\text {base }}$ and air, which is assumed to have a zero material stiffness tensor:

$$
\mathbf{C}_{i}=\boldsymbol{\alpha}_{i} \mathbf{C}_{\text {base }} .
$$

To ensure numerical stability, the minimum of $\boldsymbol{\alpha}_{i}$ is set to $\alpha_{\min }=$ $10^{-5}$. This interpolation scheme follows the established SIMP (solid isotropic material with penalization) approach for an exponent of 1 [Sigmund 2009]. Choosing a different exponent would help to converge to a binary solution in topology optimization problems with extremal objectives, where adding more material improves the objective and the maximum amount of material is fixed by a constraint. However, we do not have such an objective, and have to resort to other means to reach a binary solution. As a consequence, the exponent we choose does not influence the convergence.

The number of activations can be reduced by exploiting symmetries of the goal material. For example, for a cubic material, the response along each axis has to be identical. Mirroring the activations along all axes and all diagonal planes will therefore not constrain the solution.

\subsection{Problem Formulation}

We pose the problem of finding a microstructure that exhibits a large-scale behavior identical to a homogeneous material with desired material parameters $\mathbf{p}_{\text {goal }}$ (see Section 4.1) as a least squares problem. From the parameters $\mathbf{p}_{\text {goal }}$, a stiffness tensor $\mathbf{C}^{\text {goal }}=$ $\mathbf{C}\left(\mathbf{p}_{\text {goal }}\right)$ can be computed. The optimization then modifies the activations $\boldsymbol{\alpha}$ such that the homogenized stiffness tensor $\mathbb{C}(\mathbf{h}(\boldsymbol{\alpha}))$, which is indirectly dependent on the activations through the harmonic displacements $\mathbf{h}(\boldsymbol{\alpha})$, matches the goal stiffness tensor as closely as possible:

$$
\begin{array}{lll}
\min _{\boldsymbol{\alpha}} & \left\|\mathbf{C}^{\text {goal }}-\mathbb{C}(\mathbf{h}(\boldsymbol{\alpha}))\right\|_{F}^{2}+R \\
\text { s.t. } & \alpha_{\min } \leq \boldsymbol{\alpha}_{i} \leq 1 & 1 \leq i \leq m .
\end{array}
$$

Here, $R$ is a combined regularization term that penalizes less desirable results. This formulation differs from most other microstructure optimization approaches that typically try to find extremal properties for a specific amount of material. It is related to the formulation in [Zhou and Li 2008], though it does not use a volume fraction constraint.

\subsection{Regularization}

While the optimization problem (9) could be solved without any regularization, there is no guarantee that the result can be fabricated. In order to enforce manufacturability, we add three different regularization terms $R_{i n t}, R_{s}$ and $R_{c b}$ with corresponding weights $w_{\text {int }}, w_{s}$ and $w_{c b}$ to the objective. The combined regularization term $R$ is defined as

$$
R=w_{\text {int }} R_{\text {int }}+w_{s} R_{s}+w_{c b} R_{c b}
$$

Figure 3 and 4 show the influence of the individual regularization terms. In the following, we will elaborate on each term.

Enforcing Integer Values While the simulation allows the activations $\boldsymbol{\alpha}$ to vary freely between $\alpha_{\min }$ and 1, these configurations do not correspond to valid physical objects. For fabrication, the activations have to be integral (excluding the small offset $\alpha_{\min }$ to ensure stability). In order to reach such a solution, the regularization term $R_{\text {int }}$ acts as a penalty for activations that are not equal to either $\alpha_{\text {min }}$ or 1:

$$
R_{\text {int }}=\sum_{i=1}^{m}\left(\boldsymbol{\alpha}_{i}-\alpha_{\min }\right)\left(1-\boldsymbol{\alpha}_{i}\right) .
$$

We gradually increase the weight $w_{\text {int }}$ during the optimization, transitioning from a continuous to a discrete solution. For each value of the weight, a full optimization is run until convergence is reached. If the solution is not binary, the weight is increased and the optimization resumed. Figure 3 and the accompanying video show different stages of the optimization, with various weights $w_{\text {int }}$.

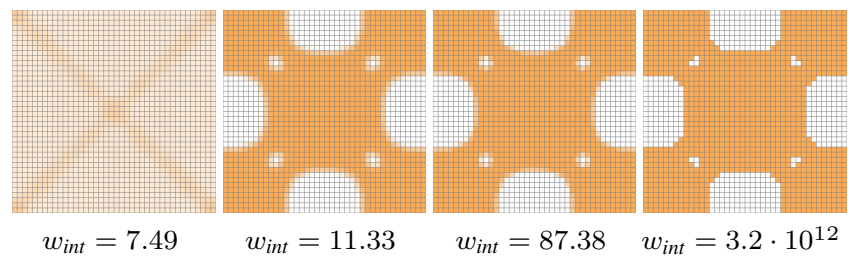

Figure 3: Optimization at different stages (with different penalty weights to force the activations towards $\alpha_{\min }$ or 1 ).

Smoothness The size of a single microstructure cell in a fabricated object is largely defined by two factors: the resolution of the 3D printer, and the size of the smallest detail in the structure. Smaller cells provide a better approximation of a continuous material, and since the printer resolution is assumed to be fixed, structures without small details are generally preferred.

The regularization $R_{s}$ views the activations as an approximation of a material distribution field, and uses a second-order finite difference approach to penalize deviations from smoothness. For this purpose, any component of $\boldsymbol{\alpha}$ is assumed to have two indices in $2 \mathrm{D}$, such that $\boldsymbol{\alpha}_{i, j}$ corresponds to the voxel $(i, j)$. The regularization then has the form

$$
\begin{gathered}
R_{s}=\sum_{i, j}\left(\boldsymbol{\alpha}_{i-1, j}+\boldsymbol{\alpha}_{i+1, j}+\boldsymbol{\alpha}_{i, j-1}\right. \\
\left.+\boldsymbol{\alpha}_{i, j+1}-4 \boldsymbol{\alpha}_{i, j}\right)^{2}
\end{gathered}
$$

in 2D; the formula for the 3D case is similar and can be found in the supplemental document. 


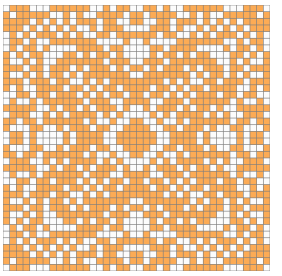

$R_{\text {int }}$

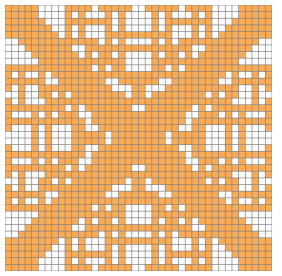

$R_{\text {int }}+R_{c b}$

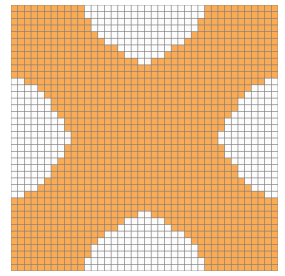

$R_{\text {int }}+R_{c b}+R_{s}$
Figure 4: Influence of different regularizations: Optimization results where only integer values are enforced (left), with additional anti-checkerboard regularization (middle) and with smoothness regularization (right). The objective value for the last two results is similar, while the first result has a worse objective value.

Checkerboard Patterns An artifact that frequently appears in topology optimization is elements that are connected by a single vertex, called checkerboard patterns [Sigmund and Petersson 1998]. To avoid such structures, the regularization $R_{c b}$ penalizes configurations that contain checkerboard patterns, as illustrated in Figure 4 . In 2D, this regularization is based on $2 \times 2$ patches of voxels and has the form

$$
\begin{aligned}
R_{c b}=\sum_{i, j} & \left(1-\boldsymbol{\alpha}_{i, j}\right)\left(\boldsymbol{\alpha}_{i+1, j}-\boldsymbol{\alpha}_{\text {min }}\right) \\
& \left(\boldsymbol{\alpha}_{i, j+1}-\boldsymbol{\alpha}_{\min }\right)\left(1-\boldsymbol{\alpha}_{i+1, j+1}\right) \\
+ & \left(\boldsymbol{\alpha}_{i, j}-\boldsymbol{\alpha}_{\min }\right)\left(1-\boldsymbol{\alpha}_{i+1, j}\right) \\
& \left(1-\boldsymbol{\alpha}_{i, j+1}\right)\left(\boldsymbol{\alpha}_{i+1, j+1}-\boldsymbol{\alpha}_{\min }\right) .
\end{aligned}
$$

In the case of binary activations, $R_{c b}$ is only non-zero if the structure contains a checkerboard pattern. In the continuous case, the regularization also acts as an additional regularizer that pushes the activations towards $\alpha_{\min }$ or 1 .

In $3 \mathrm{D}$, the number of different local checkerboard patterns increases. The corresponding formula can be found in the supplemental document.

Regularization Weights The performance of our microstructure optimization depends on the choice of weights, and how they are updated during the optimization. For the optimization in 2D, we start with $w_{i n t}^{0}=0, w_{s}^{0}=2$ and $w_{c b}^{0}=0$. Once the optimization converged for the current weights, we update them according to $w_{i n t}^{t+1}=1.3 w_{i n t}^{t}+0.1, w_{s}^{t+1}=1.1 w_{s}^{t}+0.2$ and $w_{c b}^{t+1}=5 w_{i n t}^{t+1}$, until a final solution has been found. In $3 \mathrm{D}$, we initialize the weights with $w_{i n t}^{0}=0, w_{s}^{0}=5$ and $w_{c b}^{0}=0$, and update them using $w_{i n t}^{t+1}=1.3 w_{i n t}^{t}+0.5, w_{s}^{t+1}=1.1 w_{s}^{t}+1$ and $w_{c b}^{t+1}=5 w_{i n t}^{t+1}$.

Connectivity An additional fabrication requirement is connectivity. The optimization will generally not favor binary solutions in the absence of regularization term $R_{\text {int }}$. As the influence of this term grows with increasing $w_{\text {int }}$, the previously intermediate activations will be pushed to $\alpha_{\min }$ or 1 , and the structure might become disconnected (see Figure 5). To prevent the optimization from converging to such a solution, disconnected components are detected after every iteration. To account for the continuous nature of the activations, every activation below a threshold of 0.1 is considered inactive during the detection. If a disconnected component has been found, we compute the cost of connecting the component as the smallest change in activations that builds a connection, assuming that we set the activations to a value of 0.6 . If this cost is smaller than the change in activations necessary to remove the disconnected

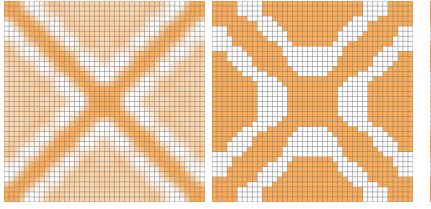

(a)

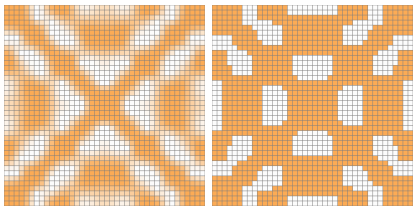

(c) (d)
Figure 5: Influence of the connectivity enforcement: Without using any connectivity enforcment during the optimization (a), the final structure might consist of several disconnected components (b). Enforcing connectivity with our scheme locally adjusts the activations (c) such that the final result is guaranteed to be fully connected (d).

component, we create the connection, and remove the disconnected component otherwise. The final result is then guaranteed to be connected.

In $3 \mathrm{D}$, an additional fabrication constraint is necessary. While 3D printing can handle complex structures, most approaches rely on support material to create overhanging structures. This support material has to be removed after printing. This means that every void voxel in the structure has to be connected to the boundary of the cell. To this end, we use the same approach we used to connect components, but switch the role of void and full voxels. In practice, we did not observe any convergence problems due to these constraints.

\subsection{Numerical Methods}

The optimization problem (9) is solved with L-BFGS-B [Byrd et al. 1995], which enforces the boundary constraints. Additionally, the indirect relationship between the coarsened stiffness tensor $\mathbb{C}(\mathbf{h}(\boldsymbol{\alpha}))$ and the activations $\boldsymbol{\alpha}$ through Numerical Coarsening has to be taken into account when computing the gradient of the objective. We refer to the supplemental document for details on this computation.

\section{Metamaterial Spaces}

The optimization method from the previous section is able to produce microstructures for a variety of material parameters, but may take a long time to generate a desired structure. Moreover, if the desired object contains spatially varying parameters, several optimizations need to be performed to generate the required microstructures, making this approach infeasible in practice. To avoid this problem, we use a data-driven approach to assemble a structure with a desired behavior by interpolation from a pre-computed metamaterial family.

A metamaterial family is a collection of microstructures, each labeled with its corresponding coarse-scale material parameters, which are a point in the space of possible material properties (parameter space); we think of the microstructure as being "located at" that point. In this section, we first describe how the structures are represented and how their locations in parameter space are determined. We then propose a technique to interpolate between these structures, providing a way to efficiently compute a corresponding microstructure for any point in the parameter space. Finally, we discuss how to use the microstructure optimization approach described in the previous section to create metamaterial families by computing sets of related microstructures that cover a wide range in parameter space.

In Section 7 we explain how several such continuous families (col- 
lectively, a metamaterial space) are used together to assemble models spanning the entire range of achievable material properties.

\subsection{Microstructure Representation}

Geometry While the voxel-based discretization we used during the microstructure optimization is useful in that context, it poses two problems for the construction of a metamaterial space: (i) The changes to the structure are all discrete in nature, so the resulting interpolation cannot be continuous, and (ii) the resulting geometry can contain stair structures. These sharp corners can lead to localized stresses under deformation and the structure would fracture more easily.

Instead of using voxels, we use signed $L_{2}$-distance fields in $[0,1]^{d}$ to represent structures in a metamaterial space, which allows for a smooth interpolation. Additionally, we perform a Gaussian smoothing step every time we sample a microstructure from the metamaterial space, which removes unwanted discretization artifacts (Figure 6). To increase resolution, we scale the grid resolution by a factor of 2 and 3 compared to the original voxelization, in 2D and $3 \mathrm{D}$, respectively.

Material Parameters Numerical Coarsening is used to compute a stiffness tensor that describes the behavior of a particular microstructure. However, for sampling and interpolation we would like to use a parameter space with fewer degrees of freedom than this tensor has (6 in two dimensions, 21 in three dimensions). By considering only isotropic, cubic or orthotropic materials, the parameter space can be reduced to a subset of material tensors, while still offering enough freedom to show a large variety of deformation behaviors.

To approximate the parameters from a material stiffness tensor computed by Numerical Coarsening, a simple constrained leastsquares approach is used. Assuming a material stiffness tensor $\hat{\mathbf{C}}$ is given, the corresponding parameters are computed by solving

$$
\begin{aligned}
& \min _{p}\|\mathbf{C}(\mathbf{p})-\hat{\mathbf{C}}\|_{F}^{2} \\
& \text { s.t. } \quad p_{i}^{\min } \leq \mathbf{p}_{i} \leq p_{i}^{\max } .
\end{aligned}
$$

The function $\mathbf{C}(\mathbf{p})$ is defined by the choice of the material model, and $p_{i}^{\min }$ and $p_{i}^{\max }$ are the physics-based bounds on the parameters, such as the lower limit of 0 for the Young's modulus. We additionally apply a normalization to the parameters that transforms all parameters with dimensions, such as the Young's modulus or the shear modulus, into dimensionless parameters by dividing them by the corresponding parameter of the base material. Since we use a linear material model, the resulting parameters describe the ratio of the structure's parameter to the base material's parameter, which also allows us to scale our results to materials with an arbitrary

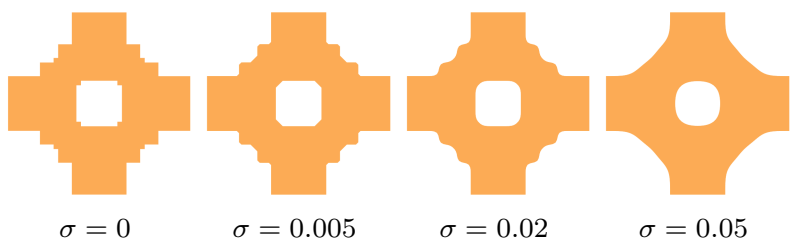

Figure 6: Results of the smoothing pass for different Gaussian spread values $\sigma$.

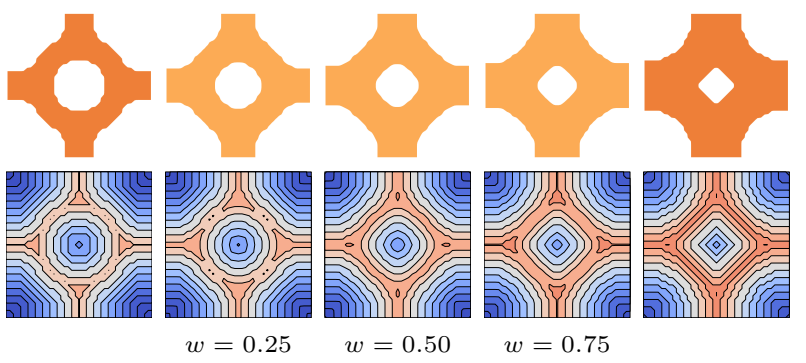

Figure 7: Top row: Results of the interpolation between the structures on the left and right for different weights $w$. Bottom row: Corresponding signed distance fields, illustrated as color plots (blue positive, red negative).

Young's modulus or shear modulus, assuming that the Poisson's ratio stays the same. Additionally, since we use this relative Young's modulus and relative shear modulus to store entries in parameter space, the distance between the parameters of two microstructures is independent of the base material's stiffness.

\subsection{Interpolation}

The microstructures in our database describe metamaterials with certain properties; each gives a point sample of the mapping from material parameters to microstructures. Figure 13, 10, and 12 illustrate data points for various metamaterial families. To generate a structure for an arbitrary given set of parameters, we interpolate between points of a family, forming a weighted average over a set of microstructures with similar elastic properties. We first compute weights based on the inverse distance between the input parameters and the parameters of the metamaterial space samples, using the Wendland function with compact support [Nealen 2004]. We chose the parameter of the Wendland function such that the weights vanish beyond a given interpolation radius, which is set to 0.1 in normalized coordinates, or the distance to the $(M+1)$ nearest neighbor ( $M$ being the number of material parameters), whichever is larger. Before we interpolate, we apply the transformation $f(x)=\operatorname{sgn}(x) \log (|x|+\delta)$ to transform the distance fields to $\log$ space, and add a small constant $\delta=10^{-3}$ to keep values near the surface. In practice, we found that interpolation in log space reduces artifacts due to topology changes, e.g., holes appearing or disappearing. Given the weights and transformed distance fields, we then compute the interpolated structure by linearly interpolating the transformed distance fields. Figure 7 illustrates the interpolation process.

\subsection{Generating Metamaterial Families}

Our metamaterial space consists of several, potentially overlapping, independent metamaterial families. We start the construction of a metamaterial family from a single microstructure, which we either model by hand based on existing examples from the literature, or obtain from our microstructure optimization (Sec. 5). We then add dilated and eroded versions of this initial microstructure to achieve a large initial sampling of the metamaterial space with good interpolation properties. The dilation and erosion is performed directly on the distance field of the structure.

The metamaterial family construction then continues to do two things: (i) Generate new candidates by evolving existing structures, which allows us to refine regions that are already covered by samples, but with insufficient sampling density, and (ii) generate new candidates by optimizing for new microstructures outside of this space. We proceed by alternating between these two stages until 
both stages do not generate new microstructures.

Evolving Existing Microstructures The first stage is a heuristic search based on the existing samples in the metamaterial family. For the refinement, we create the Delaunay triangulation of all parameter points, and collect all the simplex centers. For every center, we check how well the parameters of the interpolated structure match the desired parameters, and add the interpolated structure to the metamaterial space if the deviation from the desired parameters is too large. For our experiments, we used a threshold of 0.1 . In $3 \mathrm{D}$, we additionally try to expand the metamaterial family in this stage. For this, we first compute the convex hull of all parameter samples. For every vertex on the convex hull, we then compute an offset point along the normal, which we use as the goal parameters for a microstructure optimization. However, instead of running the optimization, we only compute the gradient of the objective and use it to change $2 \%$ of the activations in a discretized version of the current sample, which we then add to the metamaterial family.

Optimizing for New Microstructures The second stage is based on the microstructure optimization introduced in Sec. 5. For the refinement, we again compute the Delaunay triangulation and check how well the interpolation works at the simplex centers. If it is insufficient, we run a microstructure optimization for the parameters at the simplex center with an initial guess computed from the weighted combination of all samples in the neighborhood of the simplex center. Additionally, we introduce a similarity regularization, explained in more detail in the next paragraph. For the expansion, we again use the convex hull of all parameter samples and generate new parameters points by sampling the convex hull and offsetting the points along the normal. For each of these points, a microstructure optimization is run, constructing the initial guess in the same way as for the refinement and using the same additional regularization.

For any new microstructure optimization that is run for a given set of parameters, the result should be similar to the existing structure to improve the interpolation. To lead the optimization into the desired direction, an additional regularization is added to the optimization. This regularization penalizes the amount of change between a new structure and the structure of the $N$ neighbors in parameter space closest to the goal parameters. To allow for small changes, this penalty uses an exponential function:

$$
R_{\text {sim }}=\sum_{i=1}^{N} w_{\text {sim }}^{i} e^{\left(\frac{\frac{1}{m} \sum_{j=1}^{m}\left|\boldsymbol{\alpha}_{j}-\boldsymbol{\alpha}_{j}^{i}\right|}{\Delta \alpha_{i}}\right)^{2}}
$$

where $w_{s i m}^{i}=\left(N\left(1+d_{i}\right)\right)^{-1}$ is the weight for neighbor $i$ with distance $d_{i}$ to the desired parameters, $\boldsymbol{\alpha}_{j}^{i}$ is its $j$-th activation and $\Delta \alpha_{i}=0.1+2 d_{i}$ is a threshold for the maximal desirable difference to this neighbor. Additionally, the initial activations for the optimization are set to a weighted average of the neighbors, after a smoothness step is applied. This reduces the risk of ending up in a local minimum.

\section{Structure Synthesis}

Using the microstructure optimization and parameter space sampling methods, we can define several families of related structures that together span the feasible range of bulk material parameters (Figure 10). Synthesizing a homogeneous material volume at this stage becomes trivial: We select a family that covers the desired material behavior, compute the corresponding microstructure of the cell by interpolation as described in Sec. 6.2, and then fill the

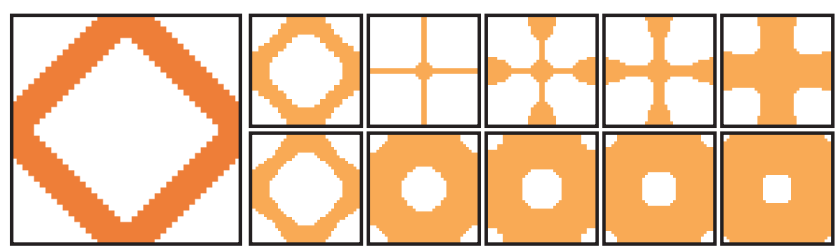

Figure 8: The first 5 microstructures generated for a parameter space without similarity regularization (top) and with similarity regularization (bottom), starting from an initial structure (left).

volume by repeating this cell. Note that by construction this cell is tileable. However, approximating spatially varying materials is more challenging.

Simply synthesizing microstructures for cells independently at each point in the model could lead to a mismatching boundary when multiple different cells are tiled, as illustrated in Figure 9. Such boundary mismatches will change the behavior of the cell, which was assumed to be in an infinite tiling of identical structures when its coarsened material parameters were computed. Therefore, both the geometry as well as the force profiles occurring at the boundaries under deformation need to be taken into account. We propose a strategy that takes advantage of the multiple candidate structures for each cell provided by the overlapping families in our metamaterial space. To compute an optimal selection from these candidates, we propose to minimize boundary dissimilarity between each pair of neighboring structures.

For a set of cells with desired parameters $\mathbf{p}_{1}, \ldots, \mathbf{p}_{k}$ and information about the connectivity between cells, we interpolate one structure for each cell from each of the $l$ families, resulting in structures $S_{i, 1}, \ldots, S_{i, l}$ for every parameter sample $\mathbf{p}_{i}$. Finding the optimal choice of structures can then be formulated as a labeling problem: assign a structure to each cell to minimize a given cost function.

We propose a cost function that combines two types of costs. The labeling cost $T_{i, j}^{L}=e^{d_{i, j}}$ describes how well a given structure $S_{i, j}$ matches the desired parameters, and is based on the distance $d_{i, j}$ between $\mathbf{p}_{i}$ and the material parameters of the structure as computed by Numerical Coarsening.

The second cost $T_{(i, j),(r, s)}^{D}=e^{g_{(i, j),(r, s)}}$ is based on the dissimilarity $g_{(i, j),(r, s)}$ of the boundaries of two neighboring structures $S_{i, j}$ and $S_{r, s}$. In most cases, it is sufficient to use the percentage of the boundary on which the two structures agree about the presence or absence of material. But since the problem of matching boundaries is linked to force discrepancies along the boundary during deformation, this dissimilarity can be improved by also considering the forces acting across the boundary. For this, we impose a unit strain

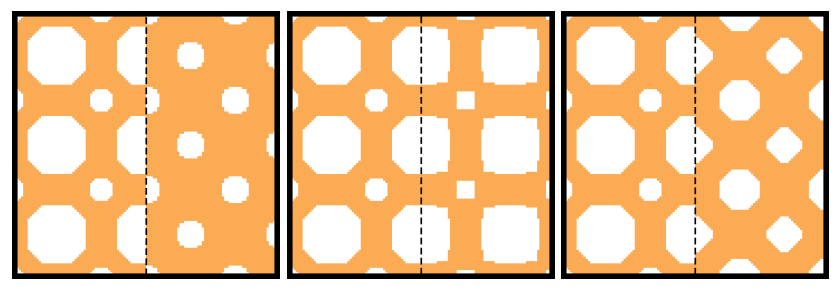

Figure 9: Three potential configurations of two neighboring cells. Left: The individual structures closely match the desired parameters, but the boundaries are incompatible. Middle: Opposite case. Right: Our optimization computes a trade-off between the two extremes. 


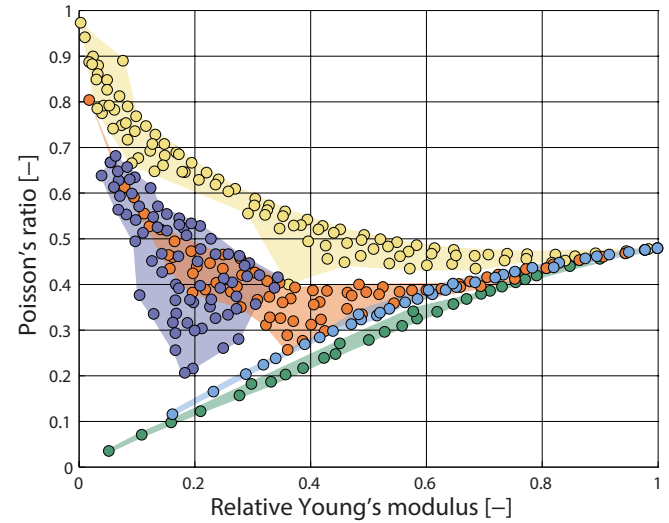

Figure 10: Data points for five different metamaterial families for a cubic material in 2D. The values for the shear modulus are omitted.

on the boundary of each cell, such that they are stretched perpendicular to the boundary between the two cells. We then integrate the force magnitude as well as the force difference magnitude over the boundary, and set the dissimilarity $g_{(i, j),(r, s)}$ to the ratio of force difference magnitude to mean force magnitude. We compare the two approaches to compute the boundary dissimilarity in Sec. 8.2.

Finding the globally optimal solution to this optimization problem is NP-hard. However, efficient algorithms exist that can find an approximate solution. We employ an iterative method using message passing based on the alternating direction method of multipliers (ADMM) as described in Derbinsky et al. [2013].

For the resulting structures, the distance fields can then be combined. To improve connectivity between cells, the smoothing pass is performed on the combined distance field instead of each cell individually. The final structure is reconstructed from the combined distance field using marching cubes.

Connectivity Our synthesis method does not guarantee connectivity. While we did not encounter cases of disconnected cells in our result, they can be detected easily and fixed by introducing additional connections between disconnected structures, at the expense of the accuracy of the approximated elastic properties.

\section{Results}

\subsection{Metamaterial Space Construction}

We tested our method on three different material classes. In 2D, we generated metamaterial spaces for cubic materials ( 3 parameters) and orthotropic materials (4 parameters), using a resolution of $40^{2}$ voxels for the microstructure optimization. Due to the inherently anisotropic nature of square and cubic microstructures, we found that a cubic material space is better suited even for cases where one is only interested in the Young's modulus and Poisson's ratio defining an isotropic material.

Figure 10 shows the Young's modulus and Poisson's ratio of multiple metamaterial families for a cubic material, and Figure 11 shows the individual families as well as a subset of their structures. While a single family may span a wide range of parameters, this example shows that combining multiple families can significantly expand this range. Figure 12 shows the orthotropic metamaterial families, projected into four different combinations of the parameter axes.

We also used our method to compute a metamaterial space for cubic

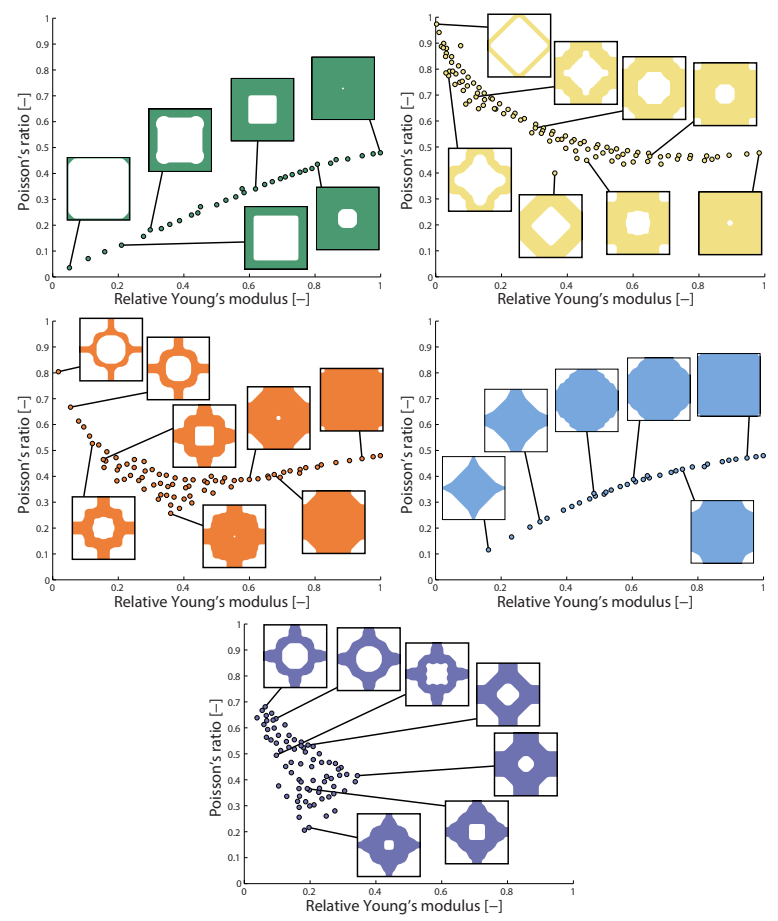

Figure 11: The individual spaces from Figure 10, including a visualization of some of the structures.

materials in 3D (Figure 13), using a $16^{3}$ voxels for the microstructure optimization.

Timings For the microstructure optimization in $2 \mathrm{D}$ with a resolution of $40^{2}$, a single optimization step takes around 200 to $800 \mathrm{~ms}$ to compute. The optimization usually converges in less than 500 iterations, resulting in a total computation time of several minutes per optimization. An optimization in 3D with a resolution of $16^{3}$ runs with 8 to 30 seconds per iteration, and usually also converges in less than 500 iterations, for a total computation time of around 2 hours per structure. Note that the metamaterial space construction is a pre-process that is only run once to build the database, and it can be easily parallelized.

For the structure synthesis, we tested the runtime of the optimization by combining three metamaterial spaces for different numbers of cells. For 400 cells, the optimization takes about 0.5 seconds, for 2500 cells about 2 seconds and for 10000 cells on the order of 40 seconds. Synthesizing the distance fields and running the optimization took less than 10 seconds in all of our examples.

\subsection{Validation}

Our method relies on the ability to compute the material behavior of a microstructure from its design. To validate the results obtained by Numerical Coarsening, we tested several of our structures in a tensile test, and compared the results to our prediction. Since we are dealing with linear elasticity, the Numerical Coarsening and the optimization itself are independent of the Young's modulus of the base material. The result can be adapted to any material with the same Poisson's ratio by a simple scaling, meaning that the ratio of the computed Young's modulus of the microstructure and the Young's modulus of the base material is constant.

Our samples were fabricated by selective laser sintering of an elastic thermoplastic polyurethane (TPU 92A-1). 


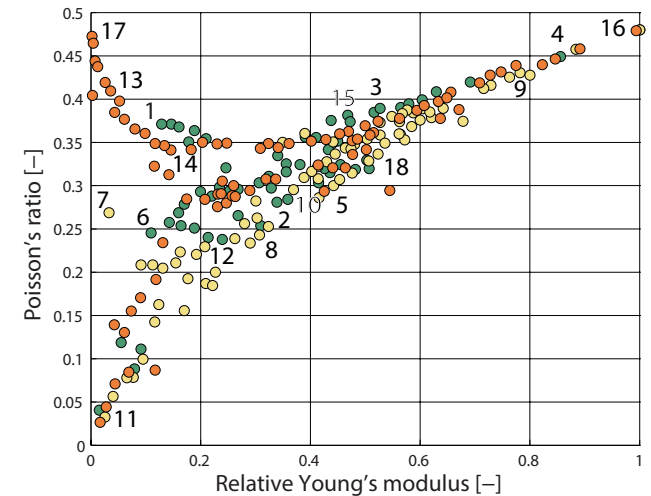

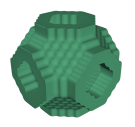
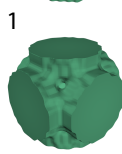

2

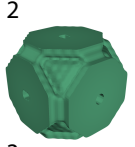

3

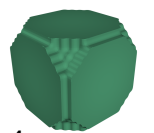

4

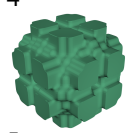

5

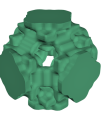

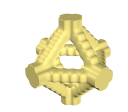

7

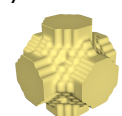

8

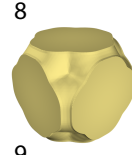

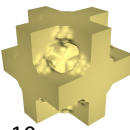

10

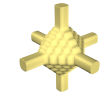

11

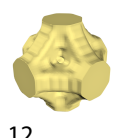

12

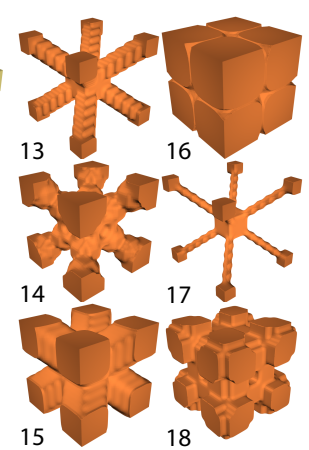

Figure 13: Data points for three different metamaterial families for a cubic material in 3D. The values for the shear modulus are omitted. A set of six structures for every family is visualized. The position of the structure is marked by the corresponding number.
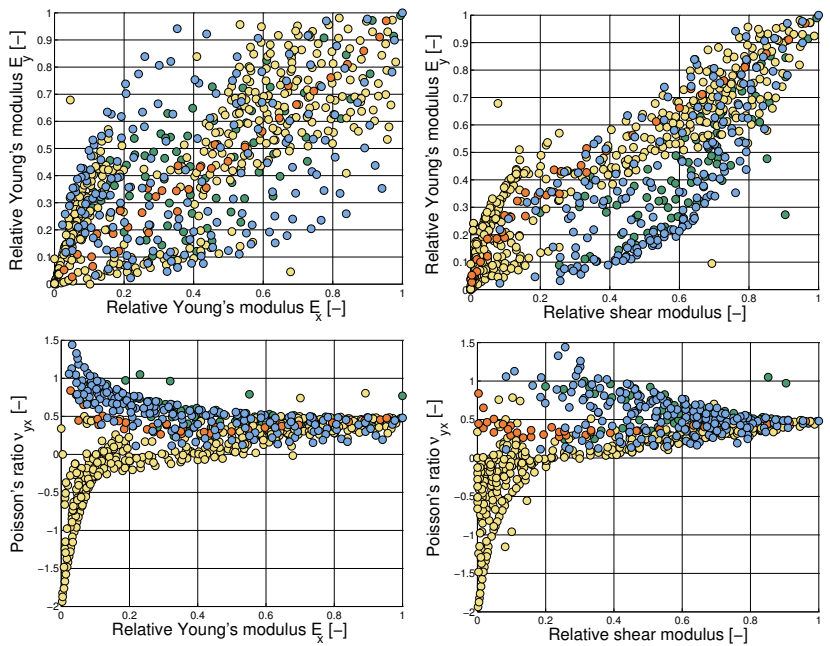

Figure 12: Data points for four different metamaterial families for an orthotropic material in $2 D$. We show the relative Young's moduli $E_{x}$ and $E_{y}$ the $x$-direction and $y$-direction, the relative shear modulus and the Poisson's ratio $\nu_{y x}$ that describes the contraction in $x$-direction for an extension applied to the y-direction.

Test Setup and Method We used an Instron E3000 frame with a $5 \mathrm{kN}$ load cell for the material test. For the 2D structures, we performed tensile tests using a $10 \mathrm{~cm}$ pneumatic grip (Figure 14). We fist characterized the base material using dog-bone shaped structures. To measure the tensile strength of the microstructures, we created samples consisting of a grid of $7 \times$ 15 unit cells $(56 \mathrm{~mm} \times 120 \mathrm{~mm})$, to reduce boundary effects. After clamping, the samples were slightly prestreched $(<1 \mathrm{MPa})$ and tested with a constant displacement rate of $50 \mathrm{~mm} / \mathrm{min}$. The three-dimensional structures were tested in a compression test, using a displacement rate of $5 \mathrm{~mm} / \mathrm{min}$. The compressive properties of the base material were measured on a cylindrical sample, and the microstructure samples used a grid of $7 \times 7 \times 6$ cells $(56 \mathrm{~mm} \times 56 \mathrm{~mm} \times 48 \mathrm{~mm}$, images in Table 1).

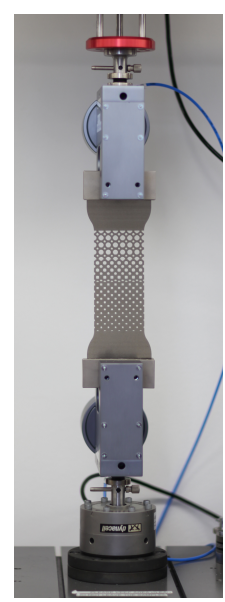

Figure 14: The test setup.
Tensile Test Results In Figure 15 we compare the measured tangent modulus vs. strain for each generated structure with a measurement of the base material scaled by the relative Young's modulus predicted by simulation. The results indicate a good fit for small strains, and for most structures even for larger strains up to 0.1 . The softest structure (9.9\% of the base material's Young's modulus) deviates slightly from the scaled base material for larger strains, showing a nearly linear behavior as compared to the nonlinear behavior of the base material. This difference is likely due to the more pronounced rotations in sparse structures.

Compression Test Results We determined the Young's modulus of the base material and three generated structures by fitting a line to the linear region of the stress-strain measurement during a loading phase. Table 1 shows that the predicted and measured relative Young's modulus match reasonably well. The stress-strain plots as well as the linear fits can be found in the supplemental document.
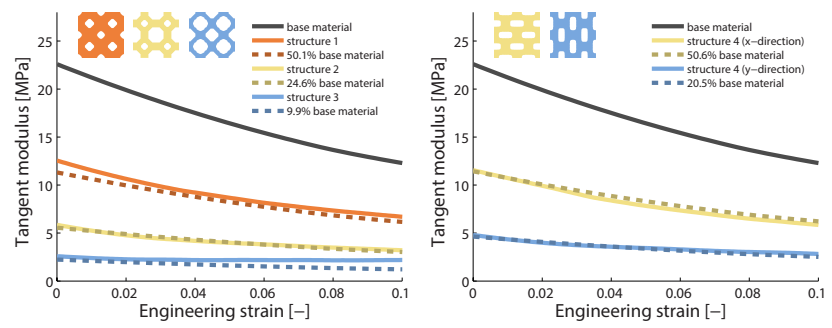

Figure 15: Tensile test results for a number of microstructures. Top: Test results for synthesized microstructures with a Young's modulus of $9.9 \%, 24.6 \%$ and $50.1 \%$ of the base material. The scaled curve of the base material is shown for reference. Bottom: Test results for interpolated microstructures with orthotropic material behavior. The computed Young's moduli were $20.5 \%$ and $50.6 \%$ of the base material's Young's modulus.

Material Gradient We tested our structure synthesis on a simple material gradient example (Figure 16) to study how well the coarsened properties match the goal for inhomogeneous cases. We specified the relative Young's modulus $E$ and Poisson's ratio $\nu$ for a $5 \times 15$ grid with a linear transition from $E=0.1$ and $\nu=0.6$ to $E=0.9$ and $\nu=0.4$, and ran our algorithm with different settings and input. Using only structures from a single family resulted in good matching of interfaces between cells, with an average boundary dissimilarity below $5 \%$. However, the average normalized dis- 


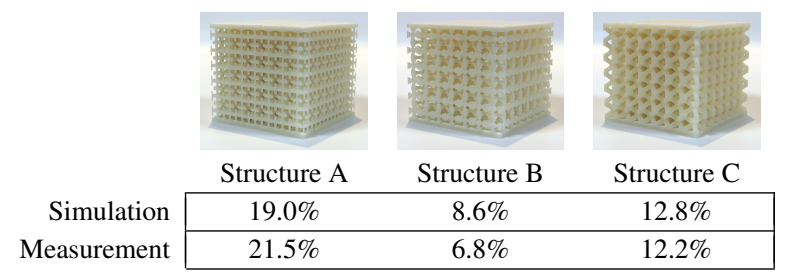

Table 1: The predicted and measured Young's moduli for each of the three structures measured in a compression test.

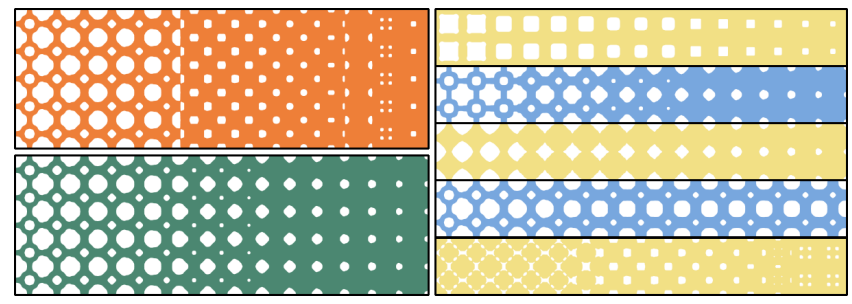

Figure 16: A material gradient with a greedy tiling (top left), showing several suboptimal tile boundaries, and our optimized tiling (bottom left). Using only one of five metamaterial spaces would result in a better tiling, but worse approximation of the desired material parameters (right).

tances between the simulated parameters of the generated structures and the desired parameters were large, with values of $0.203,0.075$, $0.177,0.228$, and 0.104 for the five metamaterial families we used for this test. By using these metamaterial families to get a set of candidate structures, we can greedily choose the best structure for each cell to achieve an average normalized distance of 0.051 . However, there is no guarantee that these structures fit together, and Figure 16 (orange) shows that the greedy synthesis generates poorly fitting transitions, with an average boundary dissimilarity of $15.3 \%$. Using the method described in Sec. 7, we can optimize for parameter approximation and boundary similarity at the same time. While the approximation of the desired parameters for the individual cells is slightly worse than for the greedy solution, with an average normalized distance of 0.065 , the boundary dissimilarity in the resulting structure (Figure 16 (green)) was significantly better (4.2\%).

Boundary Forces We presented two different approaches to represent the boundary dissimilarity during the synthesis stage. Figure 17 shows a situation in which simply comparing the geometry of the boundary performs worse than comparing the boundary forces under unit strain. We used numerical coarsening to determine the material parameters of these structures, as well as the parameters of a uniform mesh where each region is assigned the coarsened material parameters of the corresponding cells in the structure. If only the geometries of the boundaries are compared, the distance between the actual parameters of the structure and the predicted parameters is 0.0266 , while the error is only 0.0137 if the boundary forces are used to predict the dissimilarity of the cell boundaries.

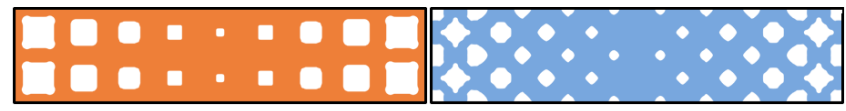

Figure 17: Two gradients computed for the same goal parameters, using geometry (left) and boundary forces (right) to estimate the boundary dissimilarity during synthesis. The actual material parameters for the right structure deviate less from the predicted parameters (error of 0.0266 , left, and 0.0137 , right).
Effects of Heterogeneity We explored our method's performance for strongly heterogeneous goal parameters with two different tests, looking at the influence of the spatial frequency and amplitude of parameter changes on the prediction error. On a grid of $12 \times 12$ cells, we synthesized structure patterns using sinusoids to define the goal Young's moduli. We tested frequencies from a single period across the grid to 6 periods (i.e. a checkerboard), and amplitudes of parameter change ranging up to $45 \%$, with a mean of $50 \%$ of the base material (so stiffnesses range from 5\% to $95 \%$ at the highest amplitude). We computed the difference between the homogenized material parameters of the synthesized structure and the homogenized material parameters of a uniform mesh with local material parameters set to the goals for the individual cells. Even though the assumption of infinite homogenous tilings is violated, Figure 18 shows that for such heterogeneous tilings, the prediction error is below 0.05 even for very drastic parameter changes, and only above that in extreme cases where the change from maximal to minimal parameters happens in a span of less than two cells, or the amplitude is larger than 0.35 .
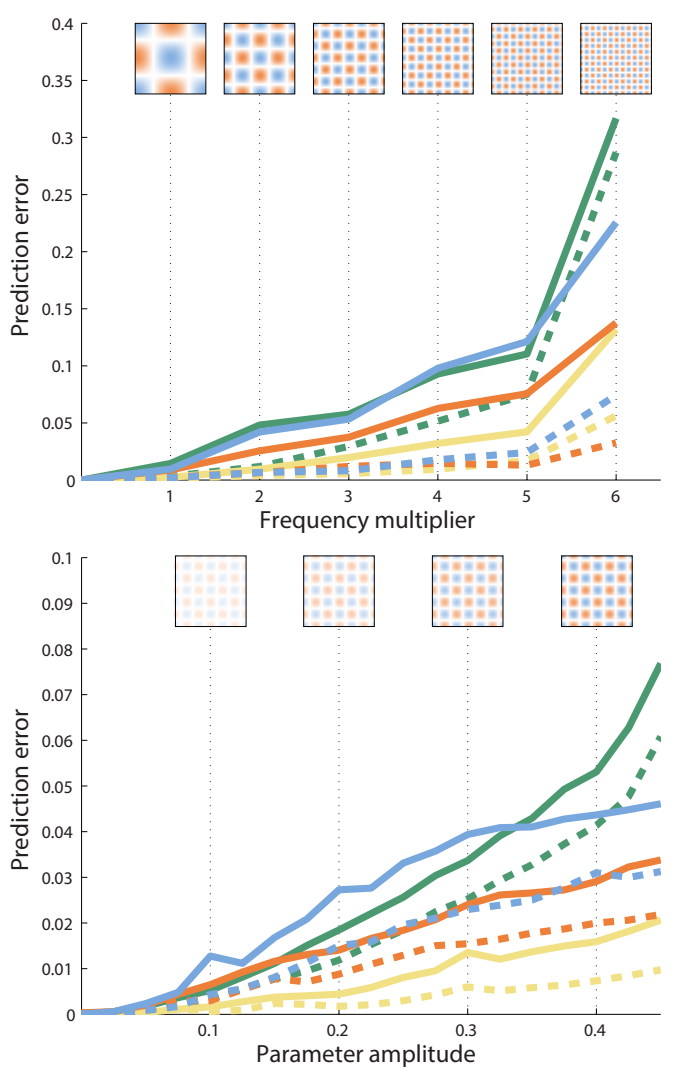

Figure 18: The influence of a heterogeneous tiling on the approximation error, based on a $12 \times 12$ grid with a sinusoidal distribution of Young's moduli. The colors of the plot match the metamaterial families shown in Figure 10. We also show the spatial distribution of the Young's moduli at the top of the plots (blue=soft, orange=stiff). Top: The error plotted for varying numbers of periods for the parameter distribution, with an amplitude of $45 \%$ (solid line) and 20\% (dashed line). A frequency multiplier of 6 corresponds to a checkerboard distribution. Bottom: The error plotted for different amplitudes, with a frequency multiplier of 3 (solid line) and 2 (dashed line). 


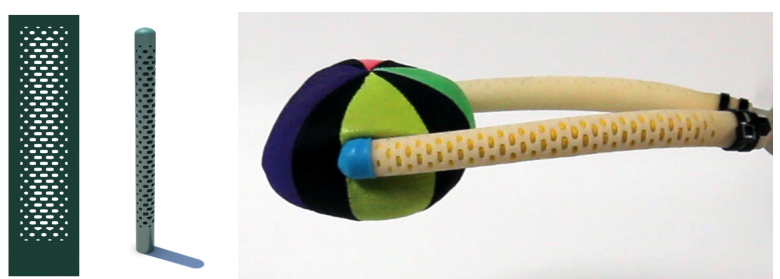

Figure 19: The optimized structure for the gripper (left), the generated model (middle). The fabricated result (right) can be used to grab and lift small objects.

\subsection{Application Examples}

Gripper Inspired by the field of soft robotics, we designed a simple gripper that can be actuated by air pressure (Figure 19). The gripper consists of two hollow tubes $16 \mathrm{~cm}$ in length, printed with a soft material. The tubes are designed as a $2 \mathrm{D}$ structure with a stiff material on one half and an anisotropic material on the other half that is soft along the tube and stiff along the circumferential direction. A balloon is inserted into each tube, and increasing the pressure inside the balloons causes the tubes to bend due to the difference in stiffness. At the same time, the anisotropy of the structure prevents large changes in diameter. While this is only a very simple actuator, we believe that our method will be an important step towards a design tool for printable soft robots.

Bunny, Teddy, and Armadillo For the three-dimensional case, we tested our pipeline on two models (Bunny, $13 \mathrm{~cm}$ high; Teddy, $15 \mathrm{~cm}$ ) with spatially varying Young's moduli, created with an interactive material design tool [Xu et al. 2015]. The models were subdivided into cells with $8 \mathrm{~mm}$ side length, and the Young's moduli averaged for each cell. The metamaterial space used to populate these cells contained a single family of 21 microstructures. For synthesis, we chose the nearest neighbor in the database for each Young's modulus. To keep the shape of the models, the individual voxels of each structure were set to void if they lay outside of the model. While this might lead to disconnected components in the reconstruction, these can easily be removed. We created a third model (Armadillo, $32 \mathrm{~cm}$ high) by manually painting the desired Young's modulus into a volumetric mesh, which was then used as an input to our method, using cells with $8 \mathrm{~mm}$ side length. We chose the parameter distribution such that the joints and the belly of the Armadillo are soft, while all other parts of the model are stiff. The structure of the cells were computed and tiled using our synthesis algorithm with the metamaterial space shown in Figure 13. The fabricated model can be easily actuated, as shown in the accompanying video, even though the base material is quite stiff.

\section{Conclusion}

We have presented a complete framework for automatically converting a given object with specified elastic material parameters into a fabricable representation that resembles the desired elastic deformation behavior. Our approach efficiently generates smallscale structures that obtain their elastic bulk properties from the shape and arrangement of the structures, significantly expanding the gamut of materials reproducible by $3 \mathrm{D}$ printers. Although our approach relies on an extensive pre-computation phase for generating families of related structures that can be interpolated to smoothly vary the material properties, this only needs to be done once. We plan to publicly release our database of structures. To create an object with spatially varying elastic properties, our approach tiles the object's interior with microstructures drawn from the database, us- ing an efficient algorithm to select compatible structures for neighboring cells.

Limitations and Future Work Our method targets output devices that can 3D print at high resolution, and that allow easy removal of support material. In practice, we found selective laser sintering the most convenient process because the part is surrounded by unsintered powder and therefore does not require support structures. Removal of the unsintered powder from the structures can be easily achieved with compressed air. Other technologies, such as polyjet or fused deposition modeling, allow printing overhangs without support structures only up to a certain angle. For future work, an interesting avenue could be incorporating these constraints into the optimization of material structures, spanning a space of metamaterials that are printable without support on these machines.

In our current work we focused on linear elasticity and small strain deformations. In the future, we plan to extend our approach to nonlinear material behavior, also incorporating interesting structures with buckling behavior. Another highly interesting avenue would be the detection of localized stresses and considering points of failure in the material structure during the optimization, improving the robustness of the material.

Finally, in our current implementation we do not explicitly treat the boundaries of the object, and obtain the boundary by simply intersecting the geometry of the structures with the shape of the object. An interesting next step would be wrapping the object with a surface for aesthetic reasons, but also taking the surface's effect on the deformation into account.

\section{Acknowledgments}

We would like to thank the reviewers for their insightful comments, and Jernej Barbič for providing models and material parameter distributions for the Bunny and Teddy examples. We also greatly appreciated the help of Moritz Bächer, Jonathan Yedidia, Mélina Skouras, and Oliver Wang. The Bunny and Armadillo models are part of the Stanford Computer Graphics Laboratory's 3D Scanning Repository.

\section{References}

ATTARD, D., AND GRIMA, J. N. 2012. A three-dimensional rotating rigid units network exhibiting negative poisson's ratios. physica status solidi (b) 249, 7, 1330-1338.

Babaee, S., Shim, J., Weaver, J. C., Patel, N., AND BERTOLDI, K. 2013. 3d soft metamaterials with negative poisson's ratio. Advanced Materials 25, 5044-5049.

Becker, M., And Teschner, M. 2007. Robust and efficient estimation of elasticity parameters using the linear finite element method. In Proceedings of the Conference on Simulation and Visualization (SimVis), 15-28.

Bickel, B., B Ächer, M., Otaduy, M. A., Lee, H. R., PfisTER, H., Gross, M., AND Matusik, W. 2010. Design and fabrication of materials with desired deformation behavior. $A C M$ Trans. Graph. (Proc. SIGGRAPH) 29, 3.

Byrd, R. H., Lu, P., Nocedal, J., AND Zhu, C. 1995. A limited memory algorithm for bound constrained optimization. SIAM J. Sci. Comput. 16, 1190-1208.

Chen, D., Levin, D. I. W., Didyk, P., Sitthi-Amorn, P., AND MatusiK, W. 2013. Spec2Fab: A reducer-tuner model 
for translating specifications to 3D prints. ACM Trans. Graph. (Proc. SIGGRAPH) 32, 4.

Cioranescu, D., And Donato, P. 2000. Introduction to homogenization.

Coelho, P., Fernandes, P., Guedes, J., And Rodrigues, H. 2008. A hierarchical model for concurrent material and topology optimisation of three-dimensional structures. Structural and Multidisciplinary Optimization 35, 2, 107-115.

Derbinsky, N., Bento, J., Elser, V., And Yedidia, J. S. 2013. An improved three-weight message-passing algorithm. arXiv:1305.1961

Dong, Y., Wang, J., Pellacini, F., Tong, X., And Guo, B. 2010. Fabricating spatially-varying subsurface scattering. $A C M$ Trans. Graph. (Proc. SIGGRAPH) 29, 4.

Hašan, M., Fuchs, M., Matusik, W., Pfister, H., And RUSINKIEWICZ, S. 2010. Physical reproduction of materials with specified subsurface scattering. ACM Trans. Graph. (Proc. SIGGRAPH) 29, 4.

HAShin, Z., AND Shtrikman, S. 1963. A variational approach to the theory of the elastic behaviour of multiphase materials. Journal of the Mechanics and Physics of Solids 11, 2, 127-140.

Hiller, J., AND LipSON, H. 2012. Automatic design and manufacture of soft robots. Robotics, IEEE Transactions on 28, 2, 457-466.

Huang, X., Radman, A., And XIE, Y. 2011. Topological design of microstructures of cellular materials for maximum bulk or shear modulus. Computational Materials Science 50, $6,1861-1870$.

Hughes, T., Marmier, A., And Evans, K. 2010. Auxetic frameworks inspired by cubic crystals. International Journal of Solids and Structures 47, 11.

Kharevych, L., Mullen, P., Owhadi, H., and Desbrun, M. 2009. Numerical coarsening of inhomogeneous elastic materials. ACM Trans. Graph. (Proc. SIGGRAPH) 28, 51.

LAKES, R. 1987. Foam structures with a negative poisson's ratio. Science 235, 4792, 1038-1040.

Lan, Y., Dong, Y., Pellacini, F., And Tong, X. 2013. Biscale appearance fabrication. ACM Trans. Graph. (Proc. SIGGRAPH) 32, 4.

Lee, J.-H., Singer, J. P., And Thomas, E. L. 2012. Micro/nanostructured mechanical metamaterials. Advanced Materials $24,36,4782-4810$.

Michel, J., Moulinec, H., And Suquet, P. 1999. Effective properties of composite materials with periodic microstructure: a computational approach. Computer methods in applied mechanics and engineering 172, 1, 109-143.

Nealen, A., Müller, M., Keiser, R., Boxerman, E., AND CARLSON, M. 2006. Physically based deformable models in computer graphics. Computer Graphics Forum 25, 4, 809-836.

NeALEN, A. 2004. An as-short-as-possible introduction to the least squares, weighted least squares and moving least squares methods for scattered data approximation and interpolation. Tech. rep., TU Darsmstadt.

Nesme, M., Kry, P. G., JeŘÁb Ková, L., And Faure, F. 2009. Preserving topology and elasticity for embedded deformable models. ACM Trans. Graph. (Proc. SIGGRAPH) 28, 3.
Reiner, T., Carr, N., Mech, R., Stava, O., Dachsbacher, C., AND Miller, G. 2014. Dual-color mixing for fused deposition modeling printers. Computer Graphics Forum (EUROGRAPHICS 2014 Proceedings) 33, 2.

Rodrigues, H., Guedes, J., And Bendsoe, M. 2002. Hierarchical optimization of material and structure. Structural and Multidisciplinary Optimization 24, 1, 1-10.

Rouiller, O., Bickel, B., Kautz, J., Matusik, W., And AleXA, M. 2013. 3d-printing spatially varying brdfs. Computer Graphics and Applications, IEEE 33, 6.

SifAKIS, E., AND BARBIC, J. 2012. Fem simulation of 3d deformable solids: a practitioner's guide to theory, discretization and model reduction. In ACM SIGGRAPH 2012 Courses, 20.

Sigmund, O., AND Petersson, J. 1998. Numerical instabilities in topology optimization: a survey on procedures dealing with checkerboards, mesh-dependencies and local minima. Structural optimization 16, 1, 68-75.

Sigmund, O. 2009. Systematic design of metamaterials by topology optimization. In IUTAM Symposium on Modelling Nanomaterials and Nanosystems, Springer, 151-159.

Skouras, M., Thomaszewski, B., Coros, S., Bickel, B., AND Gross, M. 2013. Computational design of actuated deformable characters. ACM Trans. Graph. (Proc. SIGGRAPH) 32,4 .

Smit, R., Brekelmans, W., And Meijer, H. 1998. Prediction of the mechanical behavior of nonlinear heterogeneous systems by multi-level finite element modeling. Computer Methods in Applied Mechanics and Engineering 155, 1, 181-192.

Stava, O., Vanek, J., Benes, B., Carr, N., And MĚch, R. 2012. Stress relief: improving structural strength of $3 \mathrm{~d}$ printable objects. ACM Trans. Graph. (Proc. SIGGRAPH) 31, 4.

VidimčE, K., WANG, S.-P., RAgan-Kelley, J., AND MaTUSIK, W. 2013. Openfab: A programmable pipeline for multimaterial fabrication. ACM Trans. Graph. (Proc. SIGGRAPH) 32 .

WANG, W., WANG, T. Y., YANG, Z., LiU, L., Tong, X., Tong, W., Deng, J., Chen, F., And LiU, X. 2013. Cost-effective printing of $3 \mathrm{~d}$ objects with skin-frame structures. ACM Trans. Graph. (Proc. SIGGRAPH Asia) 32, 6.

Weyrich, T., Peers, P., Matusik, W., And Rusinkiewicz, S. 2009. Fabricating microgeometry for custom surface reflectance. ACM Trans. Graph. (Proc. SIGGRAPH) 28, 3.

XU, H., LI, Y., CHEN, Y., AND BARBIČ, J. 2015. Interactive material design using model reduction. ACM Trans. Graph. (TOG) $34,2$.

Zhou, S., AND LI, Q. 2008. Design of graded two-phase microstructures for tailored elasticity gradients. Journal of Materials Science 43, 15, 5157-5167.

Zhou, Q., PAnetTa, J., And Zorin, D. 2013. Worst-case structural analysis. ACM Trans. Graph. (Proc. SIGGRAPH) 32, 4. 


\title{
Microstructures to Control Elasticity in 3D Printing - Supplemental Material
}

\author{
Christian Schumacher ${ }^{1,2} \quad$ Bernd Bickel $^{1,3} \quad$ Jan Rys $^{2} \quad$ Steve Marschner $^{4} \quad$ Chiara Daraio $^{2} \quad$ Markus Gross $^{1,2}$ \\ ${ }^{1}$ Disney Research Zurich $\quad{ }^{2}$ ETH Zurich $\quad{ }^{3}$ IST Austria $\quad{ }^{4}$ Cornell University
}

\section{Numerical Coarsening}

We use a homogenization method to describe the coarse-scale behavior of a microstructure, and as the basis of our microstructure optimization. Such a method computes material parameters for a homogenous material that approximates a structure. In the following, we summarize the Numerical Coarsening approach [Kharevych et al. 2009] and highlight differences due to its application to microstructures.

Harmonic Displacements To describe the deformation behavior of the microstructure, a set of representative displacements have to be computed for different load cases. These harmonic displacements $\mathbf{h}_{a b}$ (see Figure 1 for an illustration) are defined as the solution to the following boundary value problem:

$$
\begin{array}{llrl}
\nabla \cdot \boldsymbol{\sigma}\left(\mathbf{h}_{a b}\right) & =0 & & \text { inside } \Omega \\
\boldsymbol{\sigma}\left(\mathbf{h}_{a b}\right) \cdot \mathbf{n}=\frac{1}{2}\left(\mathbf{e}_{a} \mathbf{e}_{b}^{T}+\mathbf{e}_{b} \mathbf{e}_{a}^{T}\right) \cdot \mathbf{n} & & \text { on } \partial \Omega .
\end{array}
$$

Here, $\mathbf{e}_{a}$ is the unit vector along the $a$-th coordinate direction, $\frac{1}{2}\left(\mathbf{e}_{a} \mathbf{e}_{b}^{T}+\mathbf{e}_{b} \mathbf{e}_{a}^{T}\right)$ describes the tractions on the surface $\partial \Omega$ of the object domain $\Omega$, and $\mathbf{n}$ is the surface normal. For tiled structures, this surface is the boundary of the cell.

Considering symmetries, there are 3 and 6 distinct harmonic displacements in 2D and 3D, respectively. From these displacements, a 4-th order deformation tensor $\mathbf{G}$ can be defined per element:

$$
\mathbf{G}_{k l a b}=\left(\varepsilon\left(\mathbf{h}_{a b}\right)\right)_{k l} .
$$

This tensor contains the Cauchy strain for every displacement, and by considering the elasticity equation $W=\varepsilon: \mathbf{C}: \varepsilon$ as a bilinear equation, the term $\mathbf{G}^{T}: \mathbf{C}: \mathbf{G}$ describes the energy density for any pair of harmonic displacements.

Coarsening The homogenized material stiffness tensor can then be computed from the deformation behavior of the microstructure. The deformation is first transferred from the harmonic displacements of the microstructure to a coarse mesh consisting of only a single voxel of the size of the cell. For the case where the corners of the cell correspond to vertices of the fine mesh, this simply means transferring the displacements at the corner. For the general case, the displacement is transferred by computing a distance-weighted interpolation of a set of nearest neighbors in the fine mesh, while
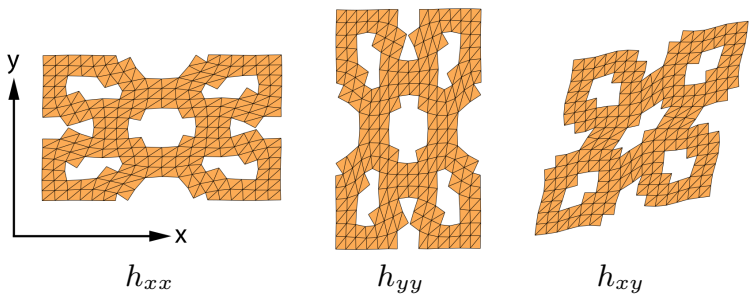

Figure 1: Harmonic displacements of a microstructure cell in $2 D$. adhering to the periodic boundary conditions. After the deformation has been transferred to the coarse mesh, a single coarse-scale deformation tensor $\mathbb{G}$ can be defined in a manner similar to Equation (2). The coarsened material stiffness tensor for the coarse mesh is then obtained analytically as

$$
\mathbb{C}=\mathbb{G}^{-T}:\left(\sum_{i=1}^{k} \frac{V_{i}}{V} \mathbf{G}_{i}^{T}: \mathbf{C}_{i}: \mathbf{G}_{i}\right): \mathbb{G}^{-1},
$$

where in the 2D (3D) case $V_{i}$ is the area (volume) of element $i$ in the fine mesh, and $V$ is the area (volume) of the entire cell. This approach differs slightly from Kharevych et al. [2009], where a coarsened material stiffness tensor is computed for every element in a coarse tetrahedral mesh. Due to the periodic boundary condition for microstructures, the strain in the coarse mesh is uniform, and a single material stiffness tensor can be computed for the whole cell. Note that we use the Voigt compressed matrix representation to express all tensors. This is especially important when computing $\mathbb{G}^{-1}$, which can be computed as a simple matrix inverse instead of a more complex symmetric tensor inverse.

\section{Microstructure Optimization in 3D}

This section describes the changes necessary to transform the microstructure optimization described in Section 5 of the paper from $2 \mathrm{D}$ to $3 \mathrm{D}$.

\subsection{Regularization}

Two of the regularization terms, the smoothness term and the checkerboard term, rely on neighborhood information and have to be adapted accordingly in a $3 \mathrm{D}$ environment.

Smoothness The approach for the smoothness term in 3D is identical to the $2 \mathrm{D}$ case, except that we now use six neighbors instead of four to compute the second-order finite difference approximation. Assuming each component of $\boldsymbol{\alpha}$ is associated with three indices in 3D, such that $\boldsymbol{\alpha}_{i, j, k}$ corresponds to the voxel $(i, j, k)$, the regularization has the form

$$
\begin{aligned}
R_{s}=\sum_{i, j, k} & \left(\boldsymbol{\alpha}_{i-1, j, k}+\boldsymbol{\alpha}_{i+1, j, k}+\boldsymbol{\alpha}_{i, j-1, k}\right. \\
& +\boldsymbol{\alpha}_{i, j+1, k}+\boldsymbol{\alpha}_{i, j, k-1}+\boldsymbol{\alpha}_{i, j, k+1} \\
& \left.-6 \boldsymbol{\alpha}_{i, j, k}\right)^{2}
\end{aligned}
$$

Checkerboard patterns In 3D, checkerboard patterns include structures that are connected by a single vertex or a single edge. To cover these two cases, we will split the regularization term in two components, $R_{c b, v}$ and $R_{c b, e}$, respectively.

To check for structures connected by a single vertex, $R_{c b, v}$ has to cover patches of $2 \times 2 \times 2$ voxels. For these patches, there are four configurations that are undesirable and will not be covered by $R_{c b, e}$. These are the only configurations for which a binary solution 
should lead to a regularization value larger than 0 . This condition can be formulated as

$$
\begin{aligned}
R_{c b, e}=\sum_{i, j, k} & \left(\boldsymbol{\alpha}_{i, j, k}-\boldsymbol{\alpha}_{\min }\right)\left(1-\boldsymbol{\alpha}_{i+1, j, k}\right) \\
& \left(1-\boldsymbol{\alpha}_{i, j+1, k}\right)\left(1-\boldsymbol{\alpha}_{i, j, k+1}\right) \\
& \left(1-\boldsymbol{\alpha}_{i+1, j+1, k}\right)\left(1-\boldsymbol{\alpha}_{i+1, j, k+1}\right) \\
& \left(1-\boldsymbol{\alpha}_{i, j+1, k+1}\right)\left(\boldsymbol{\alpha}_{i+1, j+1, k+1}-\boldsymbol{\alpha}_{\min }\right) \\
+ & \left(1-\boldsymbol{\alpha}_{i, j, k}\right)\left(\boldsymbol{\alpha}_{i+1, j, k}-\boldsymbol{\alpha}_{\min }\right) \\
& \left(1-\boldsymbol{\alpha}_{i, j+1, k}\right)\left(1-\boldsymbol{\alpha}_{i, j, k+1}\right) \\
& \left(1-\boldsymbol{\alpha}_{i+1, j+1, k}\right)\left(1-\boldsymbol{\alpha}_{i+1, j, k+1}\right) \\
& \left(\boldsymbol{\alpha}_{i, j+1, k+1}-\boldsymbol{\alpha}_{\min }\right)\left(1-\boldsymbol{\alpha}_{i+1, j+1, k+1}\right) \\
+ & \left(1-\boldsymbol{\alpha}_{i, j, k}\right)\left(1-\boldsymbol{\alpha}_{i+1, j, k}\right) \\
& \left(\boldsymbol{\alpha}_{i, j+1, k}-\boldsymbol{\alpha}_{\min }\right)\left(1-\boldsymbol{\alpha}_{i, j, k+1}\right) \\
& \left(1-\boldsymbol{\alpha}_{i+1, j+1, k}\right)\left(\boldsymbol{\alpha}_{i+1, j, k+1}-\boldsymbol{\alpha}_{\min }\right) \\
& \left(1-\boldsymbol{\alpha}_{i, j+1, k+1}\right)\left(1-\boldsymbol{\alpha}_{i+1, j+1, k+1}\right) \\
+ & \left(1-\boldsymbol{\alpha}_{i, j, k}\right)\left(1-\boldsymbol{\alpha}_{i+1, j, k}\right) \\
& \left(1-\boldsymbol{\alpha}_{i, j+1, k}\right)\left(\boldsymbol{\alpha}_{i, j, k+1}-\boldsymbol{\alpha}_{\min }\right) \\
& \left(\boldsymbol{\alpha}_{i+1, j+1, k}-\boldsymbol{\alpha}_{\min }\right)\left(1-\boldsymbol{\alpha}_{i+1, j, k+1}\right) \\
& \left(1-\boldsymbol{\alpha}_{i, j+1, k+1}\right)\left(1-\boldsymbol{\alpha}_{i+1, j+1, k+1}\right)
\end{aligned}
$$

Structures that are connected by a single edge can be detected by looking at patches of $2 \times 2 \times 1$ voxels, similar to the regularization in $2 \mathrm{D}$. The only

$$
\begin{aligned}
& R_{c b, v}=\sum_{i, j, k}\left(1-\boldsymbol{\alpha}_{i, j, k}\right)\left(\boldsymbol{\alpha}_{i+1, j, k}-\boldsymbol{\alpha}_{\min }\right) \\
&+\left(\boldsymbol{\alpha}_{i, j+1, k}-\boldsymbol{\alpha}_{\min }\right)\left(1-\boldsymbol{\alpha}_{i+1, j+1, k}\right) \\
&+\left(\boldsymbol{\alpha}_{i, j, k}-\boldsymbol{\alpha}_{\min }\right)\left(1-\boldsymbol{\alpha}_{i+1, j, k}\right) \\
&+\left(1-\boldsymbol{\alpha}_{i, j+1, k}\right)\left(\boldsymbol{\alpha}_{i+1, j+1, k}-\boldsymbol{\alpha}_{\min }\right) \\
&+\left(1-\boldsymbol{\alpha}_{i, j, k}\right)\left(\boldsymbol{\alpha}_{i+1, j, k}-\boldsymbol{\alpha}_{\min }\right) \\
&+\left(\boldsymbol{\alpha}_{i, j, k+1}-\boldsymbol{\alpha}_{\min }\right)\left(1-\boldsymbol{\alpha}_{i+1, j, k+1}\right) \\
&+\left(\boldsymbol{\alpha}_{i, j, k}-\boldsymbol{\alpha}_{\min }\right)\left(1-\boldsymbol{\alpha}_{i+1, j, k}\right) \\
&\left(1-\boldsymbol{\alpha}_{i, j, k+1}\right)\left(\boldsymbol{\alpha}_{i+1, j, k+1}-\boldsymbol{\alpha}_{\min }\right) \\
&+\left(1-\boldsymbol{\alpha}_{i, j, k}\right)\left(\boldsymbol{\alpha}_{i, j+1, k}-\boldsymbol{\alpha}_{\min }\right) \\
&\left(\boldsymbol{\alpha}_{i, j, k+1}-\boldsymbol{\alpha}_{\min }\right)\left(1-\boldsymbol{\alpha}_{i, j+1, k+1}\right) \\
&+\left(\boldsymbol{\alpha}_{i, j, k}-\boldsymbol{\alpha}_{\min }\right)\left(1-\boldsymbol{\alpha}_{i, j+1, k}\right) \\
&\left(1-\boldsymbol{\alpha}_{i, j, k+1}\right)\left(\boldsymbol{\alpha}_{i, j+1, k+1}-\boldsymbol{\alpha}_{\min }\right)
\end{aligned}
$$

\section{Numerical Methods}

The indirect relationship between the coarsened stiffness tensor $\mathbb{C}(h(\boldsymbol{\alpha}))$ and the activations $\boldsymbol{\alpha}$ through the harmonic displacements $\mathbf{h}(\boldsymbol{\alpha})$ defined in Equation (1) has to be taken into account when computing the derivatives of the microstructure optimization problem introduced in the paper. When the chain rule is applied to this problem, the Jacobian of the harmonic displacements with respect to the activations emerges. Since these quantities are effectively linked by the solution of an elasticity problem, given the boundary tractions defined in (1), we use the adjoint method to compute the Jacobian. For this, we take the derivatives of both the minimization condition $\nabla_{\mathbf{x}} U_{e l}=0$ and the constraints $\mathbf{c}=0$ with respect to $\boldsymbol{\alpha}$ :

$$
\begin{aligned}
\frac{\partial^{2} U_{e l}}{\partial \mathbf{h}_{a b} \partial \boldsymbol{\alpha}}+\frac{\partial^{2} U_{e l}}{\partial \mathbf{h}_{a b}{ }^{2}} \frac{d \mathbf{h}_{a b}}{d \boldsymbol{\alpha}} & =0 \\
\frac{\partial \mathbf{c}}{\partial \mathbf{h}_{a b}} \frac{d \mathbf{h}_{a b}}{d \boldsymbol{\alpha}} & =0 .
\end{aligned}
$$

Solving this system of equations for the desired Jacobian $d \mathbf{h}_{a b} / d \boldsymbol{\alpha}$ requires only a single sparse matrix decomposition.

\section{Compression Test Data}
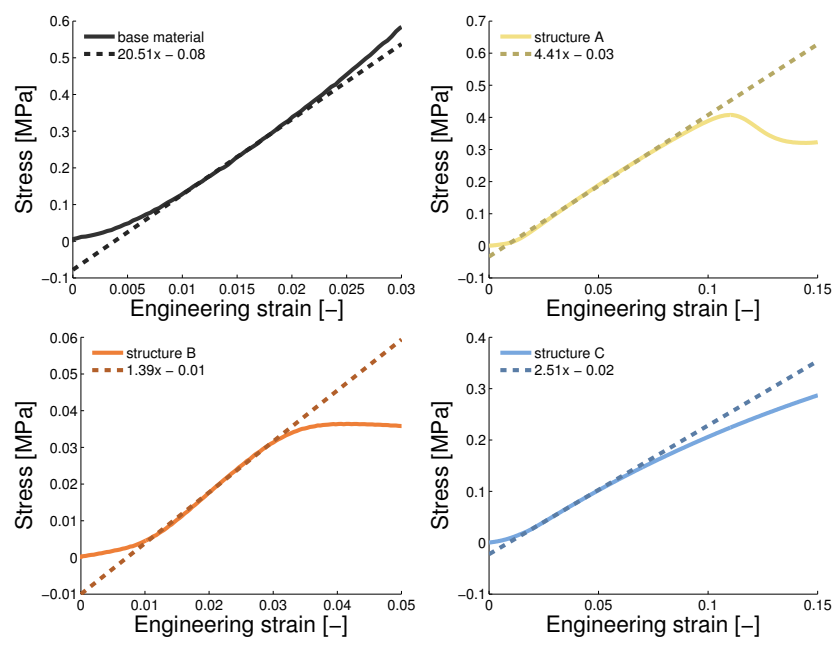

Figure 2: The stress-strain measurements for the base material and the three structures tested in the compression test. The tangents of the linear part of the curve describes the Young's modulus of the structure.

Figure 2 shows the data from the compression tests of the base material and three synthesized structure. We determined the Young's modulus of the structures by fitting a linear polynomial to the linear part of the stress-strain curve.

\section{References}

Kharevych, L., Mullen, P., Owhadi, H., And Desbrun, M. 2009. Numerical coarsening of inhomogeneous elastic materials. ACM Trans. Graph. (Proc. SIGGRAPH) 28, 51. 